A Grounded Approach for Applying Behavior Change Techniques in Mobile Cardiac Tele-Rehabilitation

Peer-reviewed author version

SANKARAN, Supraja; FREDERIX, Ines; HAESEN, Mieke; DENDALE, Paul; LUYTEN, Kris \& CONINX, Karin (2016) A Grounded Approach for Applying Behavior Change Techniques in Mobile Cardiac Tele-Rehabilitation. In: Proceedings of PErvasive Technologies Related to Assistive Environments Conference, PETRA 2016.

DOI: $10.1145 / 2910674.2910680$

Handle: http://hdl.handle.net/1942/21066 


\title{
A Grounded Approach for Applying Behavior Change Techniques in Mobile Cardiac Tele-Rehabilitation
}

\author{
Supraja Sankaran ${ }^{1}$,Ines Frederix ${ }^{2,3}$, Mieke Haesen ${ }^{1}$, Paul Dendale ${ }^{2,3}$, Kris Luyten $^{1}$, Karin Conin ${ }^{1}$ \\ ${ }^{1}$ Expertise Centre for Digital Media ${ }^{2}$ Faculty of Medical and Life Sciences \\ Hasselt University - tUL - iMinds \\ Wetenschapspark 2, \\ 3590 Diepenbeek, Belgium \\ +32- 011268411 \\ Hasselt University \\ Agoralaan - Building D \\ 3590 Diepenbeek, Belgium \\ +32- 011268111 \\ ${ }^{3}$ Department of Cardiology \\ Jessa Ziekenhuis \\ Stadsomvaart 11, \\ 3500 Hasselt, Belgium \\ +32- 011308111
}

\section{\{supraja.sankaran, mieke.haesen, kris.luyten, karin.coninx\}@uhasselt.be, ines.frederix@gmail.com, paul.dendale@jessazh.be}

\begin{abstract}
In mobile tele-rehabilitation applications for Coronary Artery Disease (CAD) patients, behavior change plays a central role in influencing better therapy adherence and prevention of disease recurrence. However, creating sustainable behavior change that holds a beneficial impact over a prolonged period of time remains an important challenge. In this paper we discuss various models and frameworks related to persuasion and behavior change, and investigate how to incorporate these with a multidisciplinary usercentered design approach for creating a mobile tele-rehabilitation application. By implementing different concepts that contribute to behavior change and applying a set of distinct persuasive design patterns, we were able to translate the high-level goals of behavior theory into a mobile application that explicitly incorporates behavior change techniques and also offers a good overall user experience. We evaluated our system, HeartHab, in a lab setting and show that our approach leads to a high user acceptance and willingness to use the system in daily activities.
\end{abstract}

\section{CCS Concepts}

- Applied computing Health care information systems - Human-centered computing Usability testing • Humancentered computing User centered design • Human-centered computing Smartphones $\cdot$ Human-centered computing Empirical studies in $\mathrm{HCI} \bullet$ Human-centered computing Ubiquitous and mobile computing design and evaluation methods - Applied computing $\sim$ Psychology $\bullet$ General and reference $\sim$ Design

Keywords

Persuasion; behavior change; tele-monitoring; tele-rehabilitation.

\section{INTRODUCTION}

After Coronary Artery Disease (CAD) patients undergo a particular treatment such as surgery, they are encouraged to go through a process of rehabilitation that can help them recover faster and reduce future risks of a similar condition. This process is commonly

Permission to make digital or hard copies of all or part of this work for personal or classroom use is granted without fee provided that copies are not made or distributed for profit or commercial advantage and that copies bear this notice and the full citation on the first page. Copyrights

for components of this work owned by others than the author(s) must be honored. Abstracting with credit is permitted. To copy otherwise, or republish, to post on servers or to redistribute to lists, requires prior specific permission and/or a fee. Request permissions from permissions@acm.org.

PETRA '16, June 29 - July 1, 2016, Corfu Island, Greece.

Copyright is held by the owner/author(s).

ACM 978-1-4503-4337-4/16/06\$15.00

DOI: http://dx.doi.org/10.1145/2910674.2910680 referred to as secondary prevention. Rehabilitation programs, part of secondary prevention, avoid or attempt to reduce the possibility of re-hospitalization [22]. However, medical studies identified a number of reasons for patient fall-outs from these rehabilitation programs. Most of the reasons cited by patients included lack of interest or motivation, geographical distance to the rehabilitation center or hospital and lack of time amidst busy lifestyle schedules [34]. As a means to overcome this, medical professionals chose to adopt tele-monitoring solutions that can help patients pursue the rehabilitation at the comfort of their home or work while being remotely monitored by care-givers at the rehabilitation center [3, 4]. In tele-rehabilitation, maybe even more than in hospital-based rehabilitation, it was observed that patients had a certain resistance to change behaviors, largely because of lack of motivation and a gradual decline in interest in the system over a period of time [29].

Employing methods of persuasive systems design could help address the behavior change challenges that exist in the context of cardiac tele-rehabilitation. However, in order to efficiently apply these methods, we need a deeper understanding of the context. Most behavior change research in the field of Human-Computer Interaction (HCI) has a focus on an individual level (such as selfdetermination and goal setting) [6-8] or on a social level (such as peer-group and social networks) and lacks addressing the broad context [6]. Also, as identified by Hekler et al. [16], in certain cases, previously developed theories of behavior change are insufficient to guide HCI research. We need additional empirical work such as qualitative approaches that can generate the knowledge necessary to establish a starting point for design. Building from various evidences on using persuasive technology to target sustained behavior change [11-13], we analyzed different behavior change theories, bridged the gap between theory and practice based on relevance to the context, identified conceptual principles that can guide the design process and applied these in the context of telerehabilitation of CAD patients. In this paper, we first set the context by describing the different persuasive theories that were considered in this context and further introduce the principles we formulated by synthesizing evidences from existing literature. We then move on to explain how we applied it in the prototype mobile application (named HeartHab) we developed, and discuss the outcomes of a usability test with the HeartHab prototype.

\section{BEHAVIOR CHANGE THEORIES FOR REHABILITATION}

To model a persuasive system to support rehabilitation, we first need to identify key behavioral patterns that foster behavioral 
maintenance and determine when and how the system should intervene [15]. For this purpose, we draw approaches from behavior change theories and user-centered design processes to strive for user acceptance as well as good support for behavior change. However, as shown by others, interpreting and using behavioral theory in order to build an interactive system is still cumbersome and challenging [7]. Current research in persuasive technology is highly theoretical and most software systems in the persuasion context are very generic and lack guidance for developers of persuasive systems to adapt them to specific contexts $[12,15,16,17]$. It remains problematic to map elements from behavior theories on suitable techniques that address the challenges of tele-rehabilitation. We combine elements from the most prevalent theories and approaches that help us to construct a comprehensive solution targeted at Coronary Artery Disease (CAD) patients. In the following sub-sections we will clarify how these selected elements are applied in the rehabilitation context for the specific target group.

\subsection{Persuasive Design Patterns}

First, in this work we consider persuasive design as a specific design approach that deals with addressing attitude and behavior change of users. As such, persuasive design can be used to incite a new behavior, modify existing behavior or reinforce current attitudes of the user [28]. To address the issue of obtaining sustained behavior change in $\mathrm{CAD}$ patients, we need persuasion tactics that can be integrated into the application and are tailored for this specific condition. We use a set of persuasive design patterns [25] that provide us with an intent and approach to support behavior change and maintenance. Apart from providing a method for presenting solutions in a structured way [20], such patterns also allow us to evaluate alternate design choices that influence patient behaviors. Based on some behaviors that needs to be targeted in our specific patient population, we selected some of these established patterns. Table 1 lists these patterns we consider in our design and classifies them into different persuasion "intents" [18, 19]. In contrast with traditional UI design patterns, persuasive design patterns still need to be mapped onto concrete UI designs. They can be considered as conceptualizations of theories that support behavior change and focus on making explicit the persuasion intent. In our current context, they guide in designing an interactive application with the intent of fostering behavior change and maintenance in $\mathrm{CAD}$ patients.

Table 1. Persuasive Design Patterns to influence behavior change in a personalized tele-rehabilitation application.

\begin{tabular}{ll} 
Persuasion intent & Design patterns \\
\hline \hline \multirow{2}{*}{ Cognition } & Illusion of control \\
& Need for closure \\
& Status quo bias \\
\hline \multirow{2}{*}{ Perception and Memory } & Reminders and Suggestions \\
& Tunneling \\
& Reduction \\
\hline \multirow{2}{*}{ Game mechanics } & Praise and Rewards \\
& Completeness meter \\
& Prolonged play \\
\hline \multirow{2}{*}{ Social role } & Credibility \\
& Coaching \\
\hline Feedback & Interaction with caregivers \\
\hline
\end{tabular}

\subsection{Models and Frameworks for Behavior Change and Maintenance}

Apart from models stemmed from psychology [21-23, 38] that have predominantly guided and formed the foundation of current research in the direction of influence and persuasion, there are numerous models that exist when it comes to behavior change support systems and persuasive technology [15, 24-27]. A comprehensive review of HCI literature on behavior change technologies identified three broad uses of behavioral theories: (a) informing the design of technical systems, (b) guiding evaluation strategies, and (c) defining target users [16]. Given our target users who are situated in the context of cardiac rehabilitation, we mainly focus on three broad theories to inform the design of HeartHab and guide its evaluation. We use the Fogg Behavior Model [7], the Persuasive Systems Design Model [26] and the Behavior Wizard Framework [10], which we will discuss in the next paragraphs.

\subsubsection{Fogg Behavior Model}

The Fogg Behavior Model (FBM) [7] is a psychological model that identifies and defines three conditions that control whether a behavior is performed: (1) motivation, (2) ability and (3) triggers. Figure 1 provides an overview of FBM with some of the persuasive design patterns (Table 1) mapped in the same space. Although these are high-level factors, FBM supports a systematic approach toward applying these factors to induce behavior change and maintenance. FBM defines that the three conditions must coincide for the behavior to occur. This framework helps to validate our persuasive design patterns by checking these conditions and whether they coincide when using these patterns. In our application, FBM conditions are translated into motivation elements for patients, simplicity of the interface and appropriate means for triggering behavior change. FBM leads to defining a common frame of reference that can be used while creating specific goals for patients. In the context of rehabilitation, small daily objectives can be easily mapped in Figure 1 above or below the green action line based on patients' specific ability and motivation to estimate the right direction in which they need to be triggered.

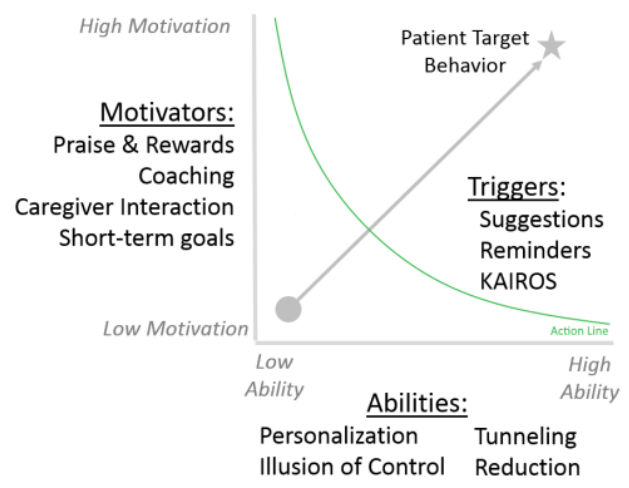

Figure 1. Selected persuasive design patterns classified as motivators, abilities and triggers; adapted from FBM [7].

\subsubsection{Persuasive Systems Design Model}

The Persuasive Systems Design Model (PSD) [26] proposes a set of principles of persuasion that can be mapped on software quality requirements, such as simplicity, responsiveness and information quality. Our selection of the various design patterns listed in Table 1 can contribute to fill this gap. The various postulates behind persuasive systems that are identified by PSD show how these map to various persuasion intents and design patterns (as represented in Table 1). These eventually lead to creating events and strategies that need to be adopted. The design guidelines mentioned in this 


\begin{tabular}{|c|c|c|c|c|c|}
\hline & $\begin{array}{c}\text { GREEN } \\
\text { Do new behavior }\end{array}$ & $\begin{array}{c}\text { BLUE } \\
\text { Do familiar behavior }\end{array}$ & $\begin{array}{c}\text { PURPLE } \\
\text { Increase behavior intensity }\end{array}$ & $\begin{array}{c}\text { GRAY } \\
\text { Decrease behavior intensity }\end{array}$ & $\begin{array}{c}\text { BLACK } \\
\text { Stop existing behavior }\end{array}$ \\
\hline $\begin{array}{l}\text { DOT } \\
\text { One Time }\end{array}$ & $X$ & $X$ & $X$ & $X$ & $X$ \\
\hline $\begin{array}{c}\square \text { SPAN } \\
\text { Period of time }\end{array}$ & $X$ & $X$ & $X$ & $\begin{array}{c}\text { CORONARY ARTERY DISEASE } \\
\text { + CARDIO MYOPATHY }\end{array}$ & $X$ \\
\hline 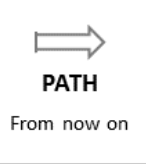 & $\begin{array}{c}\text { CORONARY ARTERY DISEASE } \\
\text { + HEART FAILURE } \\
\text { Obesity }\end{array}$ & $\begin{array}{l}\text { Type } 2 \text { diabetes } \\
\text { Dislipedemia }\end{array}$ & $\begin{array}{c}\text { CAD } \\
\text { Type } 1 \text { diabetes } \\
\text { Hypertension } \\
\text { Renal failure }\end{array}$ & $X$ & Smoking \\
\hline
\end{tabular}

Figure 2. Patient risk factors mapped into target behavior zones using the Behavior Wizard.

model are also based on Fogg's model and since our design patterns fit well in that model, it can validate the approach we adopt to build the rehabilitation system. The approach that is mentioned in PSD of categorizing postulates also gives us a well-defined structure to develop and evolve a design life-cycle.

\subsubsection{Behavior Wizard Framework}

The patient risk factors that we refer to are basically medical conditions that are related to heart disease such as obesity, diabetes hypertension and so on. Every patient tends to have one or more of these medical conditions which have ultimately led to a certain heart disease or adversely influence an existing heart condition. In rehabilitation, it is essential to target and address these causes as a primary measure of secondary prevention to minimize future risk and re-hospitalization rates. The rehabilitation process for each of these conditions vary and it is important to consider the precise combination that can work effectively for every individual patient. Consequently, the prescriptions are tailored to match specific patient risk profiles. Let us consider an example of a CAD patient who is obese. A typical rehabilitation recommendation for this kind of patient will be as follows- "The patient will have to indulge in a moderate intensity physical activity 3-5 days a week for a minimum of 60 mins per session and for at least 24 weeks." This simple statement gives us a lot of information in terms of the type of behavior (physical activity in this context) that needs to be obtained, the duration and the frequency. Thus, to map the above example using the behavior wizard, we can assert that the patient has to learn a new behavior and continue doing it from now on since the recommended duration gives only the minimum value and says that the patient must adhere to it for at least 24 weeks. Therefore, as depicted in Figure 2, such a patient can be categorized into the green-path grid. Similarly, the behavior wizard can help categorize all patients with different risk factor categories and heart conditions. This method gives us a more structured approach to design and formulate a tailored rehabilitation intervention for every individual patient category.

\section{BRIDGING THEORIES}

Applying behavior change theories for persuasive applications often requires using an ungrounded interpretation owing to lack of examples or well described good practices. According to a recent work on clarifying behavior change theories for general health applications [32], the application of these theories for rehabilitation is more critical. In the broad context of health behavior change for rehabilitation, a broad range of aspects have to be considered such as habits, the scenario, environment, ability to exercise, levels of motivation, individual patient pathologies, combinations of risk factors that can influence recurrence of disease and other factors that influence behavior to a great extent. Habits are cultivated over many years and modifying them is highly challenging. This becomes an important aspect to consider given that the average age of patients in cardiac rehabilitation is high and triggering new healthy habits therefore becomes difficult.

In most scenarios while designing for health behavior change, goals merely mention abstract targets such as "lose more weight" or "be more active". These are generalizations and not specific behavior targets. Such targets are not useful in promoting or triggering a change in behavior. Patients have to be gradually nudged to achieve their rehabilitation goals. In a diverse patient population, it is hard to specify the perfect recipe and build patterns that can guide each patient in a specific direction within a short span of time. So the key is to identify patterns that work in a similar context, try to map the target patient behavior to any of those patterns and assess the level of influence accordingly. We did this using the behavior wizard as explained in Section 2.2 (Figure 2).

Though behavioral insights may not offer a definitive solution, they can be leveraged to provide new useful evidences and frameworks that can enhance conventional rehabilitation systems in a very clear and cost-effective manner. Using the foundations of the best practices laid down by Fogg [8], we developed 8 conceptual principles in order to efficiently leverage the plethora of theories and frameworks discussed in Section 2.

\subsection{Attract}

In order to persuade someone, it is important to first catch their attention. Attract is the preliminary principle which deals with attracting patients and giving them a reason to consider a novel approach to rehabilitation. This can, for instance, be achieved by an interesting priming by doctors or rehabilitation care-givers and an attractive, user-friendly interface.

\subsection{Stimulate}

Once we catch the attention of patients in a positive manner, it is important to give them reasons to look forward to, while progressing with the rehabilitation. This principle will help to keep the patients stimulated to ensure behavior maintenance over a longer duration of time. This can be achieved by tailoring and optimizing the system based on the patients' pathological information that is monitored. Therefore, their personal information at one point of time (such as their weight) will be used to influence the perception of further information that will be presented to them (such as configuring optimal weight zone and training goals). 


\subsection{Engage}

This principle is very closely related to the principle of stimulation. Alongside stimulating them, it is essential to keep patients engaged to enable long-term adherence to the prescribed behavior change. This can be achieved by designing short-term or micro-goals. Such short term goals also help in observing evolution of patients' progress earlier than in the case of long-term goals. These little goals should be designed to be less strenuous while effectively contributing to long-term goals and benefits. This requires an ongoing commitment from patients and can thus ensure continuity.

\subsection{Maintain}

Having ensured patient-engagement, it is important to ensure there are no trivial reasons for dropout. This principle deals with the robustness of design to ensure that the system is bug-free and there are no factors that negate a good user-experience. This can be achieved by adopting validated design practices and iterative usability tests to check for flaws early in the design and development process and patch them effectively.

\subsection{Break-Down}

This principle deals with creating design chunks based on specific tasks. To assist in maintaining robustness and to build an engaging interface, it is essential to break-down complex tasks and operations into manageable units. This will not only simplify the actions to be performed by the patients but will also make it easier to keep the system updated.

\subsection{Order}

The design chunks broken down have to be organized and sequenced into logical task-flows. This principle helps in sequencing design chunks, creating logical navigational structures and prioritizing tasks and screens according to what is essential for patients. For example, the most frequently accessed screens which help in monitoring various parameters can be ordered on the top of the navigational structure and other generic screens such as patient profiles, while settings can be pushed down.

\subsection{Nudge}

This principle corresponds to providing small behavior triggers through design. These triggers can be composed in multiple ways. Triggers could be intuitive suggestions to pursue a goal, or explicit reminders and suggestions to perform a certain activity such as taking a walk. A simple medication reminder is another example of a trigger. These triggers and nudges in design will subtly persuade patients in forming habits and gradually modifying behavior in a positive direction.

\subsection{Reward}

Without sufficient incentives to drive patients, there is a possibility that all the above principles fail. This principle focuses on embedded rewards in the design for various actions performed by the patient. These rewards could be brownie-points that they gain for achieving their short-term goals, performance badges or a ranking system amidst peer-patient groups. However, the best reward for patients will be a noticeable change to healthy behavior and the improved quality of life that this system will deliver.

The above principles have been validated against some projects in our research group that were proven to contribute positively to motivational aspects of behavior change in cardiac rehabilitation. This was an informal validation done by checking the principles and their visible aspects in the designs of these projects. However, it gave a broader context and informed the current research with best practices. The application of theories to design based on the above principles has concretely been validated in the HeartHab context based on the preliminary usability test outcomes discussed in Section 5.

\section{APPLYING THEORY IN DESIGN}

We collated different persuasive design patterns that are relevant to cardiac tele-rehabilitation (as discussed in Section 2.1) and thoroughly evaluated various theories pertaining to behavior change and persuasive technology (as discussed in Section 2.2). Drawing from those inferences, we applied the conceptual principles we derived in Section 3 while designing some initial prototypes for HeartHab. To tailor the visual interface and adapt it to the specific target group more accurately, it is essential to follow a user-centered design (UCD) approach. Since a cardiac telerehabilitation system involves different users such as patients and care-givers (for HeartHab mainly doctors and physiotherapist, and a psychologist) we adopted a multi-disciplinary user-centered design approach [13]. This iterative approach can be related to the conceptual principle "maintain" (as explained in Section 3.4). This process began with a structured analysis phase in which we identified the various user goals and resources needed to perform various tasks to achieve the goal. This was done by conducting a workshop and multiple brainstorming and discussion sessions with medical practitioners (cardiologist and cardiologist in training) involved with cardiac rehabilitation at the local rehabilitation center. We decided to begin with the patient-side of the application. In the subsequent stage of the UCD process we created low-fidelity mockups of the mobile application we envisioned. After using these to drive further discussions, we came up with logical navigational structures and organized the features accordingly. The navigational structures were used in the next stage to create an interactive highfidelity prototype that was tested from a usability perspective with $\mathrm{CAD}$ patients in the particular target group.

The subsequent paragraphs will illustrate how we applied ideas of persuasive design while prototyping HeartHab, the mobile application for cardiac tele-rehabilitation. HeartHab has been developed to support the need for secondary prevention and does not replace the current rehabilitation program. It will be used as a tool to enable patients to continue and follow-up on their rehabilitation at home after the hospital-based rehabilitation is complete, or it can be used in combination with the final weeks of an ambulant rehabilitation program. The behaviors that are targeted by our application are rehabilitation adherence, continuity and long-term maintenance of healthy habits to minimize risk factors. Drawing from the design patterns listed in Section 2.1, we apply the following behavior change approaches to reach our targets.

\subsection{Reduction}

Patients suffering from cardiac illness are usually prescribed to take multiple medicines at different moments of the day. It often becomes difficult for them to remember what medicine has been taken or is to be taken. To simplify this critical task, reduction can be used to design an easy-to-use medication prescription widget. Reduction helps to reduce complex human behavior by breaking it down, and aids in increasing medication compliance.

We designed a prescription wheel (Figure 3(a)) that essentially divides the day into four quadrants and gives patients simple information on what medicine to take at which part of the day. Upon tapping on a specific quadrant, the application opens a novel prescription representation (Figure 3(b)), showing a reduced list of medicines to be taken at that period of the day and check buttons to mark after taking the specific medicine. A medicine bar can further be expanded to get more information about that medicine's side effects. This novel representation to persuade patients to comply 


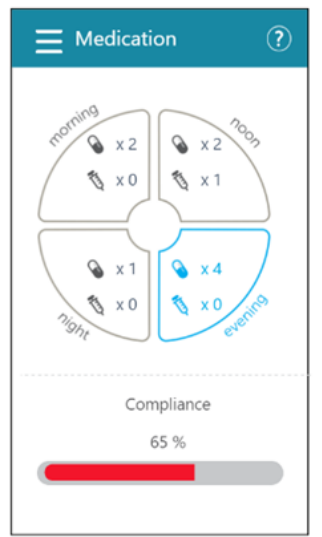

a) Medication

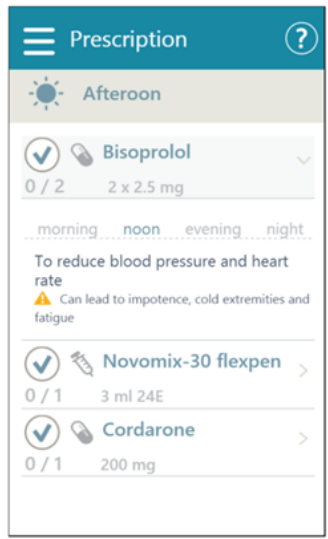

b) Prescription

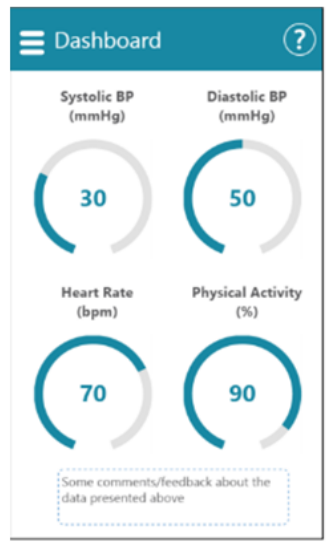

c) Dashboard

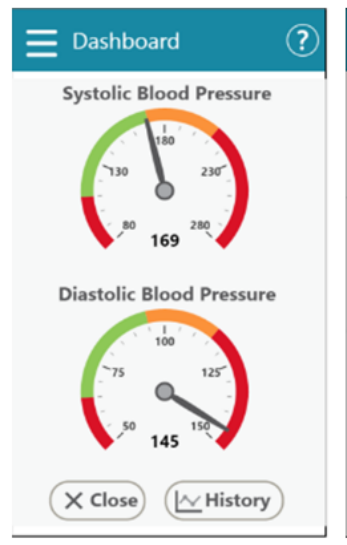

d) Dashboard- Semantic Zoom

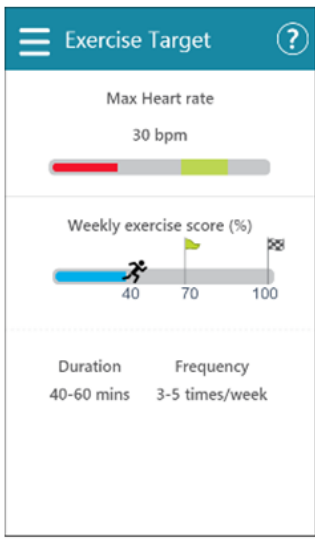

e) Exercise Target

Figure 3. Screenshots from HeartHab representing features that were tested with patients.

with medication adherence corresponds to the conceptual principle "attract" (mentioned in Section 3.1).

\subsection{Defaults}

Patients have different physiological conditions and a patient expects to see information based on their individual profile and concerns. The choice of defaults can be leveraged efficiently to deal with this situation by providing specific choice frames for every patient. A default is defined as a choice frame in which one selection is pre-selected and individuals have to take deliberate steps if they want to select a different option [20]. When it comes to common aspects such as medication reminders or triggers to motivate patients to be more active, simple and generic defaults can be framed that are applicable to all patients in general. On a more specific scope, notifications and overview displays can be optimized by creating more tailored default frames. For example, we display notifications specific to glucose for a diabetic patient or weight for an obese patient and optimize corresponding visualizations on the dashboard screen.

These default frames are constructed in a manner that can be tailored and configured for every individual patient to encourage them better as aimed by the conceptual principle "stimulate" The default configurations are decided by the medical professionals (or care-givers) based on patient physiological conditions measured by them. This method of constructing defaults by care-givers also caters to the credibility design pattern which is crucial in applications such as this.

\subsection{Status quo bias}

It has been shown in multiple studies that defaults are very powerful to guide choices $[12,13]$. However, there could be ethical risks associated with setting defaults or "forcing choice". Therefore we allow users to make a "guided choice" in a limited set of defaults that all contribute to the desired behavior change, instead of streamlining them into a single pre-constructed default. In fact this is an application of status quo bias, in which steering has a positive connotation rather than the incorrect forcing of choices to a baseline situation. Applied to HeartHab, the generic default constructs can be kept flexible to give users more freedom to customize it. For example, while performing routine tasks such as checking blood pressure or weight, the widgets on the dashboard (Figure 3(c)) can be moved around to enable patients to choose the default view. They can organize and arrange parameters that are most relevant for them. On the other hand, the option to modify default constructs for critical aspects such as configuring training goals will remain with caregivers. By giving patients the control to optimize some generic defaults and customize it according to what appeals to them more, we also intuitively cater to the illusion of control design pattern. Thus, this can form an effective means to cater to the conceptual principle "nudge" (as discussed in Section 3.7) as they can act as small behavior triggers.

\subsection{Tunneling}

In a comprehensive application that encompasses multiple features, it is often difficult to direct users' action in one direction. To avoid detours from desired behavior, tunneling can guide users through a specific process and attempt to persuade them during the course of action. With this aim of helping patients focus on one action at a time, we applied the concept of tunneling across various elements in HeartHab (correlating the conceptual principles "break-down" and "order" as detailed in Sections 3.5 and 3.6 respectively). For example, in the medication page, the patients are first represented with a simple medication wheel (Figure 3(a)) showing the amount of medicines that have to be taken at a certain time of the day. The quadrant that represents the current time of day is highlighted which acts as a subtle nudge to guide users to select the highlighted quadrant to see what actions need to be taken at that point of time. Similarly, a minimalistic dashboard representation (Figure 3(c)) helps the user get information in an easy to understand manner. If the user wishes to get more detailed information, a mere tap on a parameter pops out a detailed gauge (Figure 3(d)) that presents information such as optimal range and current value in an intuitive and color-coded manner.

\subsection{Completeness meter}

When it comes to channelizing a user's behavior towards a specific goal, it is essential to find means of communicating the end of a certain part of the process and presenting what comes next. It is also essential to provide this in a tangible manner that they can strive to achieve. Apart from catering to the conceptual principle "engage" (as explained in Section 3.3), a completeness meter can address this need for closure effectively by rewarding users with a clear representation of their goal. Giving them a sense of achievement in doing simpler things and getting rewarded for those things is much better than attempting to push them to do harder activities and rarely rewarding. Existing rehabilitation prescriptions merely present targets as numbers and patients tend to lose motivation over a period of time. In order to direct the patients' behavior towards their activity goals while keeping them motivated, we designed a gamified completeness meter (Figure 3(e)). Based on their exercise prescription, a weekly goal is computed and represented by a checkered finish flag, while their monitored progress is visualized 


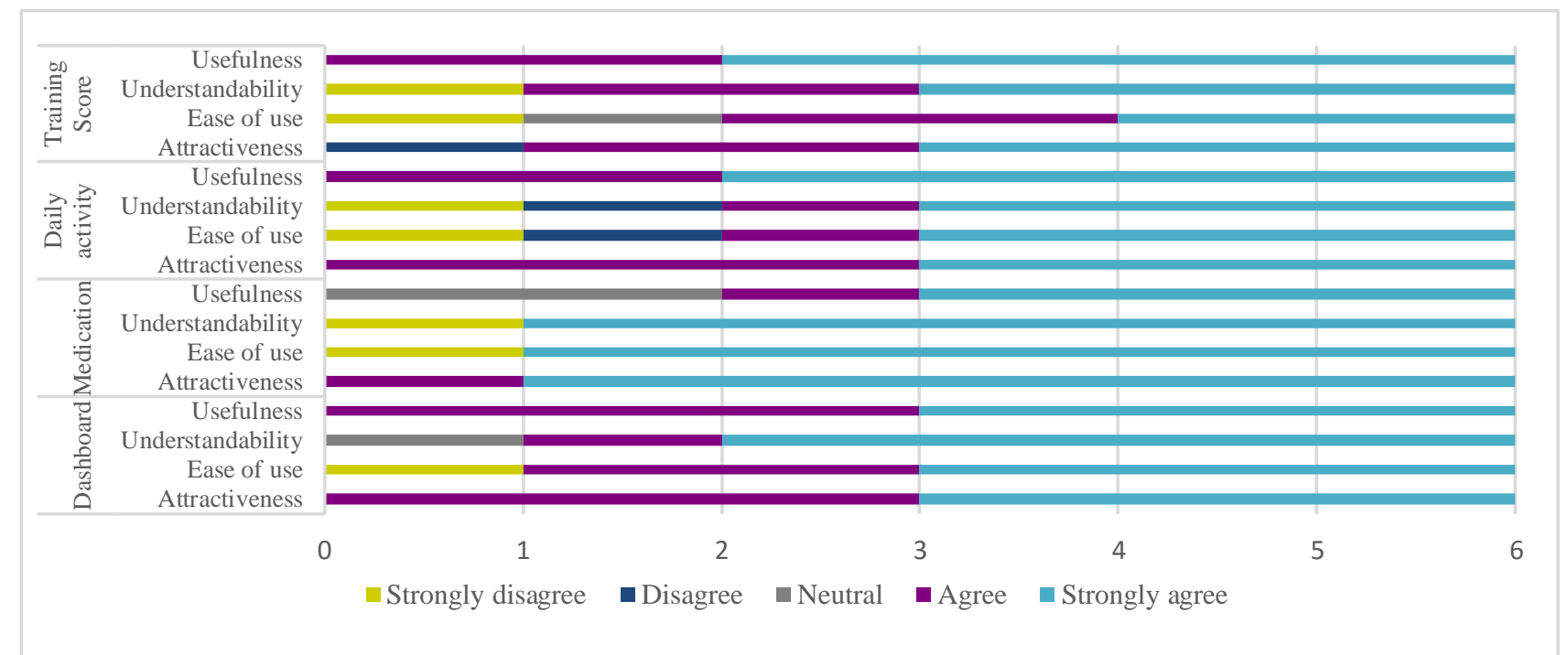

Figure 4. Summary of results of the usability tests.

using an animated person running towards the goal. As an additional nudge to keep them motivated, an optimal target zone is also marked using a green checkpoint flag.

\section{USABILITY TESTS AND OUTCOMES}

The multi-disciplinary user-centered design approach, involves a preliminary lab test to test the user experience against initial interactive high-fidelity prototypes. The goal of this study was to gain insight on some fundamental usability aspects such as patients' comfort with using mobile technology, ease of navigation through different screens of HeartHab and understandability and relevance of various features.

\subsection{Study Setup}

The study was performed in a lab setup where the smartphone running HeartHab was placed on a table. A camera was placed above the smartphone which could record participants' interactions with the application. The camera also had additional audio recording capabilities. To facilitate observation and analys is later on, the camera focused only on the screen of the smartphone. The face of the user was not captured to maintain anonymity. The test was conducted by a team consisting of one facilitator and two observers. Pilot tests were conducted with 4 researchers from our group to fine-tune the test setup.

\subsection{Methodology}

A group of six participants ( 5 males and 1 female) was recruited by a medical professional of the rehabilitation center. While three of these participants have been a part of an earlier tele-monitoring study, the other three were naïve participants. The earlier study however, did not involve mobile applications on smartphones and only focused on monitoring walking activities using a pedometer or accelerometer. The feedback system of this study was manually configured by the care-giver based on the data collected by the device.

In each individual test session, a participant was introduced to the study by the facilitator who explained the purpose of the study and the informed consent. The participant was also encouraged to "think aloud" while performing the tasks. The participant was asked to fill out a pre-test questionnaire to gauge his/her technology familiarity and exposure to different devices. To get introduced to the interaction with the smartphone and the HeartHab system, the participant was then given an exploratory task focusing on the profile page, with small steps detailed out. The main part of the test consisted of 4 tasks that were directly related to the screenshots presented in Figure 3. For each task, the participant was presented with a hypothetical situation and asked to perform two to three related subtasks. These tasks basically required the participant to navigate through various elements of the application, look for some specific information, or enter new information regarding an activity.

Finally, after completing all tasks, the patient was asked to fill out a post-test questionnaire. In the questionnaire, opinions were collected using a five-point Likert scale to evaluate the design, ease of use, understandability and usefulness of various elements. A few open questions in the end of the questionnaire collected overall remarks, suggestions and feedback.

\subsection{Results}

The questionnaires, videos and notes taken during the test were thoroughly analyzed and we arrived at the following results. The pre-test questionnaire revealed that most participants used computers or a generic mobile phone everyday while only 3 participants used a smartphone. The participants who used a smartphone, used it only for generic communication purposes such as making calls or sending text messages. While 2 patients (who were both a part of the aforementioned tele-monitoring study) used a pedometer, none of the 6 participants had any experience of using other health or fitness applications.

We observed that all participants were able to use HeartHab on the smartphone with little or no initial help. However, the various tasks performed by the participants, helped us to gather feedback for a next iteration in the UI design of HeartHab. For example, most participants had difficulties in interpreting some terminology and icons, and also mentioned problems with some fonts and colors.

The post-test questionnaire showed that most participants were comfortable with the overall design and could find information easily. Figure 4 summarizes the participants' assessments of various elements of HeartHab. The feature that got the most positive response was the novel medication prescription and reminder feature. One participant remarked: "It was logically grouped". Another key feature that all participants were in favor for was the gamified approach to target their training objective. With respect to other elements, most participants found all features interesting but a couple of them were prioritizing what they would really use and not use (often based on their own medical situation), which inspires us to provide a customizable interface in a next iteration of HeartHab. 


\subsection{Discussion}

Health behavior change through rehabilitation is a long, complex and sensitive process for the patient. Evaluating the change is challenging as well, and it adds complexity to the design process. In initial prototypes, it is not feasible to demonstrate substantial influence of novel methodologies or ideas on health behavior change. In their extensive analysis, Klasnja et al. [18] propose that a deep understanding of how and why of system use by its target users should be the central goal of evaluating systems of health behavior change. They further state that this thorough understanding of system use, and the resultant design knowledge, is arguably the biggest contribution that HCI can make to the development of effective systems in the domain of behavior change. We aim to contribute in that aspect by using our approach of bridging theories and formulating conceptual principles (as explained in Section 3) to guide the design of systems for rehabilitation of $\mathrm{CAD}$ patients.

Using this approach for prototyping helped in achieving highly positive usability responses from patients. The promising initial feedback from the usability tests also motivates us to progress further in this direction. Although initial results are only indicative, they help us gain a good understanding of different patient perceptions and their experience with technology. As stated by Oduor et al. [25], conducting such user testing offers a unique opportunity to quantify measures of system success by explaining the intent in terms of perceived behavior change goals.

This test does not give us a detailed insight into the persuasiveness of the application, as this only can be evaluated in a longer study. However, responses that show patients' interest to use it every day and their positive feedback on the gamified training goal elements show preliminary indications that this approach can work to keep patients motivated. The gamification aspect also caters to the conceptual principle "reward" (mentioned in Section 3.8).To convert this into achieving sustained behavior change, further emphasis needs to be laid on fulfilling intrinsic goals [12]. The usability tests show that we succeeded to integrate the persuasive design principles in HeartHab, whilst maintaining a good level of usability.

\section{CONCLUSION AND NEXT STEPS}

In this paper we discussed how we strive for a grounded application of behavior change techniques in mobile cardiac tele-rehabilitation. Considering various models and frameworks related to persuasion and behavior change, we investigated how to incorporate these in a mobile tele-rehabilitation application. It was deemed essential to fit the approach in a user-centered design methodology that optimally takes into account stakeholders of the application. Concretely we propose a set of distinct persuasive design patterns, facilitating the translation of the high-level goals related to persuasion and behavior change into a concrete design for the mobile application that shows good overall user experience. We reported on our labbased usability evaluation, and conclude that our approach leads to a high user acceptance and intention to use the system in daily activities.

In order to dig deeper into specific evaluation of the persuasiveness, we iterate further on the mobile application according to the multidisciplinary user-centered design approach (Section 4). Accordingly, the high-fidelity prototype has been extended with application logic, and a field test recently started. The test protocol for the field test instructs participants to use the app in daily life over a few weeks. Though we think this paper contributes in increasing the applicability of frameworks and models for persuasion and behavior change through our proposed principles, we are fully aware that evaluating their effectiveness needs further attention. In the context of behavior change support systems, selfreporting is considered the only appropriate method for measuring attitude [35]. Therefore, in the ongoing field test, we hand over the app for 4-6 weeks. A weekly journal for patients to self-report and $\log$ their experiences during the process of tele-rehabilitation is one of the evaluation techniques in this study. There is evidence that even a short study of a few weeks can reveal information regarding persuasion and behavior change. The fact that overall user goals are broken down in smaller, short-term goals (as discussed in Section 3 ) opens up the possibility to integrate these first measurements in the field test.

On the longer term, the mobile application is tested by our medical colleagues for its clinical relevance and effectiveness for cardiac tele-rehabilitation. Typical experimental designs for these studies in the medical domain are Randomized Control Trials (RCTs), which are also very suited for the evaluation of behavior change in case the study period is long enough [36, 37]. On the other hand, intervention studies are more and more accepted for early evaluations in technology-supported rehabilitation, and these might include evaluation of persuasiveness and behavior change when testing over a significant number of months. In the domain of health behavior change support systems, Portnoy et al. [5] extensively studied 75 RCTs most of which succeeded by combining multiple theories and adding motivational components. Given that our proposed approach is in line with the strategies that proved to be successful, we are looking forward to see confirmation of our approach in the current field test and future longitudinal studies on behavior change and clinical effectiveness.

\section{REFERENCES}

[1] Ajzen, I. 1985. From intentions to actions: a theory of planned behavior. Springer Berlin Heidelberg (1985), 11-39. DOI=https://dx.doi.org/10.1007/978-3-642-69746-3.

[2] Cialdini R 2007. Influence: the psychology of persuasion. HarperCollins Publishers (2007). ISBN-13:978-0061241895.

[3] Consolvo, S., Klasnja, P., Mcdonald, D.W. and Landay, J.A. 2009. Goal-Setting Considerations for Persuasive Technologies that Encourage Physical Activity. In Proceedings of Persuasive '09 ACM, NY. 8, 8. DOI=http://dx.doi.org/10.1145/1541948.1541960.

[4] Consolvo, S., Markle, K., Patrick, K. and Chanasyk, K. 2009. Designing For Persuasion: Mobile Services for Health Behavior Change. In Proceedings of Persuasive '09. ACM, NY. DOI=http://dx.doi.org/10.1145/1541948.1541964.

[5] David B. Portnoy, Lori A. J. Scott-Sheldon, Blair T. Johnson, and M.P.C. 2009. Computer-Delivered Interventions for Health Promotion and Behavioral Risk Reduction: A MetaAnalysis of 75 Randomized Controlled Trials. Prev Med. 47, 1(2008),3-16. DOI=http://doi.org/10.1016/jypmed200802014

[6] Dourish, P.2010. HCI and Environmental Sustainability: The Politics of Design and the Design of Politics. Proceedings of DIS '10. DOI=http://dx.doi.org/10.1145/18581711858173.

[7] Fogg, B. 2009. A behavior model for persuasive design. In Proceedings of the Persuasive '09. ACM, NY. DOI=http://dx.doi.org/10.1145/1541948.1541999.

[8] Fogg, B.J. 2009. Creating Persuasive Technologies: An Eight -Step Design Process. In Proceedings Persuasive '09. ACM, NY. DOI=http://dx.doi.org/10.1145/1541948.1542005.

[9] Fogg, B.J. 2009. The Behavior Grid: 35 Ways Behavior Can Change. In Proceedings of Persuasive '09. ACM, NY. 
DOI=http://dx.doi.org/10.1145/1541948.1542001.

[10] Fogg, B.J. and Hreha, J. 2010. Behavior Wizard : A Method for Matching Target Behaviors with Solutions. In Proceedings of Persuasive '10. 117-131. DOI=http://dx.doi.org/10.1007/978-3-642-13226-1_13.

[11] Frederix, I., Driessche, N. Van, Hansen, D., Berger, J., Bonne, K., Alders, T. and Dendale, P. 2013. Increasing the medium-term clinical benefits of hospital-based cardiac rehabilitation by physical activity telemonitoring in coronary artery disease patients. Eur J Prev Cardiol. 22, 2 (Feb. 2013), 150-8. DOI=10.1177/2047487313514018.

[12] Gal-oz, O.Z.A. 2014. Deconstructing gamification: evaluating the effectiveness of continuous measurement, virtual rewards, and social comparison for promoting physical activity. Pers Ubiquit Comput. 18, 7 (Oct. 2014), 1705-1719. DOI=http://dx.doi.org/10.1007s0077901407832.

[13] Haesen, M., Coninx, K., Bergh, J. Van Den and Luyten, K. 2008. MuiCSer: A Process Framework for Engineering Processes. Int Fed Info Proc. (2008), 150-165.

[14] Hansen, D., Dendale, P., Raskin, A., Schoonis, A., Berger, J., Vlassak, I. and Meeusen, R. 2010. Long-term effect of rehabilitation in coronary artery disease patients: randomized clinical trial of the impact of exercise volume. Clin Rehabil 24, 4(2010), 319-27. DOI=10.1177/0269215509353262.

[15] Hekler, E.B., Buman, M.P., Poothakandiyil, N., Rivera, D.E., Dzierzewski, J.M., Morgan, A.A., McCrae, C.S., Roberts, B.L., Marsiske, M. and Giacobbi, P.R. 2013. Exploring behavioral markers of long-term physical activity maintenance: a case study of system identification modeling within a behavioral intervention. Health Educ Behav. 40, (Oct. 2013), 51-62. DOI=10.1177/1090198113496787.

[16] Hekler, E.B., Klasnja, P., Froehlich, J.E. and Buman, M.P. 2013. Mind the Theoretical Gap: Interpreting, Using, and Developing Behavioral Theory in HCI Research. In Proceedings of CHI '13. ACM, NY, 3307-3316. DOI=http://dx.doi.org/10.114524706542466452.

[17] Johnson, E.J., Herrmann, A. and Heitmann, M. 2008. Nudge Your Customers Toward Better Choices. Harvard Business Review (2008).

[18] Klasnja, P., Consolvo, S. and Pratt, W. 2011. H ow to Evaluate Technologies for Health Behavior Change in HCI Research. In Proceedings of CHI '11. ACM, 3063-3072. DOI=http://dx.doi.org/10.114519789421979396.

[19] Locke, E. a. and Latham, G.P. 2006. New directions in goalsetting theory. Curr Dir Psychol Sci. 15, 5 (2006), 265-268. DOI=10.1111/j.14678721.2006.00449.x.

[20] Lockton, D. 2013.Design with intent: a design pattern toolkit for environmental and social behaviour change. $\mathrm{PhD}$ Theses. Brunel University School of Engineering and Design.

[21] Lockton, D., Harrison, D. and Stanton, N.A. 2010. The Design with Intent Method: A design tool for influencing user behaviour. Appl Ergon., 41, 3. 382-392. DOI=http://dx.doi.org/10.1016/j.apergo.2009.09.001.

[22] Mampuya, W.M. 2012. Cardiac rehabilitation past, present and future: an overview. Cardio diag and therapy (2012), 38-49. DOI=http://doi.org/10.3978/jissn2223365220120102.

[23] Oinas-kukkonen, H. 2013. A foundation for the study of behavior change support systems. Pers Ubiquit Comput. 17,
6 (Aug. 2013), 1223-1235.

DOI=http://dx.doi.org/10.1007/s00779-012-0591-5.

[24] Oinas-kukkonen, H. 2010. Behavior Change Support Systems: The Next Frontier for Web Science. In Proceedings of the Web Science Conf (2010).

[25] Oinas-kukkonen, H. 2014. Persuasive software design patterns for social influence. Pers Ubiquit Comput. 18, 7 (2014) 1689-1704. DOI=http://dx.doi.org/10.1007/s00779014-0778-z.

[26] Oinas-Kukkonen, H. and Harjumaa, M. 2009. Persuasive systems design: Key issues, process model, and system features. Communications of the Association for Information Systems. 24, (2009), 485-500.

[27] Oinas-kukkonen, H. and Oinas-kukkonen, H. 2010. Behavior Change Support Systems: A Research Model and Agenda. In Proceedings of Persuasive '10, 4-14. DOI=http://dx.doi.org/10.1007/978-3-642-13226-1_3.

[28] Oinas-kukkonen, H., Oinas-kukkonen, H. and Harjumaa, M. 2008. Towards Deeper Understanding of Persuasion in Software and Information Systems. In Proceedings of ACHI 08. 200-205. DOI=http://dx.doi.org/10.1109/ACHI.2008.31.

[29] Patrick D. Savage, Bonnie K. Sanderson, Todd M. Brown, K. and Berra, P.A.A. 2012. Clinical Research in Cardiac Rehabilitation and Secondary Prevention: Looking Back and Moving Forward. J Cardiopulm Rehabil 31, 6 (Nov. 2011), 333-341. DOI=10.1097/HCR.0b013e31822f0f79.

[30] Petty, R.E. 1986. The elaboration likelihood model of persuasion. Adv exp soc psychol 19, (1986), 123-205. DOI=10.1016/S0065-2601(08)60214-2.

[31] Reinwand, D., Kuhlmann, T., Wienert, J., de Vries, H. and Lippke, S. 2013. Designing a theory- and evidence-based tailored eHealth rehabilitation aftercare program in Germany and the Netherlands: study protocol. BMC public health. 13, (2013), 1081. DOI=10.1186/1471-2458-13-1081.

[32] Schneider, H., Moser, K., Butz, A. and Alt, F. 2016. Understanding the Mechanics of Persuasive System Design : a Mixed-Method Theory-driven Analysis of the Mobile Fitness Coach Freeletics. In Proceeding of CHI '16 (2016).

[33] Sunstein, C.R., Thaler, R.H. 2003. Libertarian Paternalism Is Not an Oxymoron.U Chicago Law Rev 70,(2003), 1159-1202

[34] Turk-Adawi, K.I., Oldridge, N.B., Tarima, S.S., Stason, W.B. and Shepard, D.S. 2013. Cardiac rehabilitation patient and organizational factors: what keeps patients in programs? JAHA, 2(5). DOI=http://doi.org/10.1161/JAHA.113.000418.

[35] Wiafe, I., Nakata, K. and Gulliver, S. 2014. Categorizing users in behavior change support systems based on cognitive dissonance. Pers Ubiquit Comput. 18, 7 (2014), 1617-4909.

[36] van den Berg M, Schoones J, Vliet V.T. Internet-Based Physical Activity Interventions: A Systematic Review of the Literature J Med Internet Res 9,3(2007). DOI=10.2196/jmir93.e26.

[37] Hurling R, Catt M, De Boni M, Fairley B, Hurst T, Murray P, Richardson A, Sodhi J. Using Internet and Mobile Phone Technology to Deliver an Automated Physical Activity Progrram: Randomized Controlled Trial. J Med Internet Res 9, 2 (2007). DOI=10.2196/jmir.9.2.e7.

[38] Festinger, L. 1957. A theory of cognitive dissonance Row \& Peterson. 